\section{Partial protection may encourage additional threats to MPAs}

This study applies indices to quantify MPA effectiveness in reducing anthropogenic threats (extractive and non-extractive) in and around 15 Mediterranean MPAs. The authors show that fully-protected areas effectively eliminated threats from extractive activity. However, within partially-protected areas the intensity of artisanal and recreational fishing was actually higher than that found outside MPAs. In addition, both fully and partially protected areas attracted non-extractive activities (tourism) that posed potential threats.

The term marine protected area (MPA) is fairly broad in that MPAs can have very different levels of protection. Fully-protected areas ban all extractive activities, like fishing, oil and gas drilling, and seafloor mining. Partially-protected areas - which might be better known as multiple-use MPAs - only ban certain extractive activities while allowing others.

To compare different MPAs with varying levels of protection, the authors developed a framework to quantify local potential threats to the MPAs' biodiversity, both extractive (e.g., fishing) and not (e.g., tourism). The framework relies on threat indices, including the intensity of the potential threat and the threat reduction capacity afforded by the MPA. MPAs were scored based on how well they reduced threats. A negative MPA threat reduction score indicated an MPA decreased threats. In contrast, a positive MPA threat reduction score indicated an MPA was actually encouraging or attracting threats.

A local threat index was calculated to determine the intensity of threats outside of a given MPA compared to threats outside all of the region's MPAs. These indices were aggregated and standardized for each MPA on a 0-1 scale, with higher scores indicating the given MPA was experiencing comparatively more threats.

When comparing these indices, fully-protected MPAs - as one would expect - had no extractive threats like partially-protected ones did. However, fully-protected areas had higher intensities of non-extractive threats: on average, 2.6 times the threat level of partially-protected MPAs and fully 16 times the threats of areas surrounding the MPAs. Scuba-diving and commercial boating were among the greatest non-extractive threats.

Partially-protected areas saw the highest levels of artisanal and recreational fishing: about 5 times as much as levels outside MPAs. Extractive activities were 18 times greater in intensity as non-extractive activities in areas outside MPAs.

The authors found no relationship between the size or age of an MPA and its ability to reduce threats. Nor did they find a relationship between the MPA's ability to reduce threats and the intensity of threats near it.

What can we take away from this research? Protecting areas from certain uses may encourage others. Take a city street, for example: typically, streets are shared among cars, bicycles, and pedestrians. If you ban cars from a street, then you're probably going to see more pedestrians and bicycles than you did before. So, where partially-protected areas ban commercial fishing, it logically follows that you'll see more artisanal and recreational fishing when the small guys don't have to worry about being in the way of large fishing vessels.
This is a summary of: How good is your marine protected area at curbing threats?

Accessible at: https://marxiv.org/2ry6t

Authors: Mirta Zupan, et al

Added to MarXiv:

April 2018

Published: Biological Conservation, 2018

Suggested Citation: Partial protection may encourage additional threats to MPAs. OCTO (2018). DOI: 10.17605/OSF IO/6KRNF

See more MarXiv summaries at https://www.marxivinfo.org/ summaries

Share your research in MarXiv and the MarXiv Team may summarize it, just like this!

Step-by-step instructions on how to share your research in MarXiv are detailed at https://www.marxivinfo.org/ submission

Across all disciplines, papers available for free with a preprint (also known as "Green OA") are cited $30 \%$ more than the world average (DOI: 10.7287/peerj. preprints.3119v1) 


\section{MarXiv Summary}

MarXiv: The free research repository for the ocean \& marine-climate sciences

Fully-protected areas might see more tourism-related activities, since divers don't have to worry about being fished themselves.

MPA managers can use this framework of threat indices to compare various sites and the potential regulations for those areas to ensure the threats that matter are reduced. Knowing that non-extractive activities may increase once protection is afforded can help plan for additional human impacts later on.

\section{MarXiv}

The MarXiv Team

Nick Wehner,

Director of Open Initiatives,

OCTO

Raye Evrard,

Project Manager, ОСТO

Allie Brown,

Project Associate, ОСто

Sarah Carr,

Chief Knowledge Broker,

OCTO

John Davis,

President, OCTO

Correspondence

Nick Wehner

nick@octogroup.org

Financial support for MarXiv comes from the David and Lucile Packard Foundation.

The views expressed herein are those of the MarXiv Team and should not be interpreted as representing the opinions or policies of the Packard Foundation or other funders of MarXiv.

Join the MarXiv Summaries monthly newsletter at https:// oct.to/marxivsum

MarXiv is an OCTO Initiative
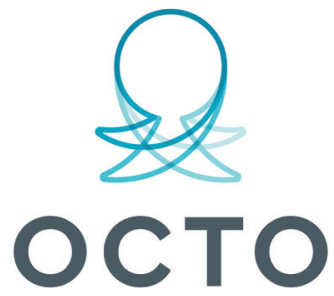

OPEN COMMUNICATIONS FOR THE OCEAN 